\title{
Impact of learning HIV status on contraceptive use in the MIRA trial
}

\author{
Kelly Blanchard, ${ }^{1}$ Kelsey Holt, ${ }^{2}$ Alan Bostrom, ${ }^{3}$ Ariane van der Straten, ${ }^{4}$ Gita Ramjee, ${ }^{5}$ \\ Guy de Bruyn, ${ }^{6}$ Tsungai Chipato, ${ }^{7}$ Elizabeth T Montgomery, ${ }^{8}$ Nancy S Padian ${ }^{9}$
}

\author{
'President, Ibis Reproductive \\ Health, Cambridge, MA, USA \\ ${ }^{2}$ Senior Project Manager, Ibis \\ Reproductive Health, Cambridge, \\ MA, USA \\ ${ }^{3}$ Specialist, University of \\ California, San Francisco, CA, \\ USA \\ ${ }^{4}$ Senior Research Scientist, \\ Women's Global Health \\ Imperative, RTI International, San \\ Francisco, CA, USA \\ Director, HIV Prevention \\ Research Unit, Medical Research \\ Council, Durban, South Africa \\ ${ }^{6}$ Programme Director for HIV \\ Prevention Studies, Perinatal HIV \\ Research Unit, Johannesburg, \\ South Africa \\ ${ }^{7}$ Professor of Obstetrics \\ and Gynecology, College of \\ Health Sciences, University of \\ Zimbabwe, Harare, Zimbabwe \\ ${ }^{8}$ Research Public Health Analyst, \\ Women's Global Health \\ Imperative, RTI International, \\ San Francisco, CA, USA \\ ${ }^{9}$ Adjunct Professor, School of \\ Public Health, University of \\ California, Berkeley, CA, USA
}

\section{Correspondence to} Kelsey Holt, Ibis Reproductive Health, 17 Dunster Street Suite 201, Cambridge MA 02138,USA; kholt@ ibisreproductivehealth.org

Received 10 August 2010 Accepted 8 April 2011 Published Online First 11 July 2011

\begin{abstract}
Background and methodology We examined the effect of becoming HIV-positive on contraceptive practices in a Phase III randomised controlled trial of the diaphragm for HIV prevention. We coded self-reported contraceptive use into seven categories of methods in order of effectiveness. We compared the proportion using each category of contraception at baseline and last visit between women who did and did not become HIV-positive. We compared changes in the proportion using each category of contraception from baseline to last visit and calculated the percentage of women that moved to a more or less effective method category or stayed the same. We examined immediate and long-term changes in contraceptive use category after learning HIV-positive status. Results A total of 4645 women remained HIV-negative and 309 became HIV-positive. The proportion using each category of contraception was similar between groups at baseline and last visit. In both groups approximately one-fifth changed to a less effective method category between baseline and last visit. Few women reported using longacting methods. Among HIV-positive women, shorter-term changes in the proportion using each category of contraception were similar to longer-term changes, though somewhat more women were using a method in the same category 3 months after seroconversion.
\end{abstract}

Discussion and conclusions Learning about HIV-positive status did not appear to significantly change patterns of use of effective contraceptives or the probability of switching to a more or less effective method. Information about, and access to, long-acting methods should receive more attention and be a routine part of family planning programmes and prevention trials.

\section{Introduction}

In sub-Saharan Africa approximately $60 \%$ of adults living with HIV are women. ${ }^{1}$ Unintended pregnancy is also a

\section{Key message points}

Learning about HIV-positive status did not appear to significantly change patterns of use of effective contraceptives or the probability of switching to a more or less effective method in this study.

- Information about, and access to, long-acting contraceptive methods should receive more attention and be a routine part of family planning programmes and prevention trials.

significant problem and 25\% of married women aged 15-49 years in the region are interested in preventing or delaying pregnancy but do not have access to contraception. ${ }^{2}$ Data on the impact of HIV-positive diagnosis on pregnancy prevention strategies can help identify gaps in education and access, informing the development of interventions to improve the quality of, and access to, services and counselling.

Few studies have documented the contraceptive practices of women in sub-Saharan Africa who have recently learned they are HIV-positive. A longitudinal study among Malawian women found a significant increase in contraceptive use within the first week of learning one's HIV-positive status, though use declined between 3 and 12 months' follow-up and ended somewhat higher than original baseline rates. ${ }^{3}$ A study evaluating prevention of mother-to-child-transmission in Kenya and Zambia found that HIVpositive women were significantly more likely to use condoms for family planning than HIV-negative women in two of the three sites, whereas use of other methods was similar between HIV-positive and HIV-negative women. ${ }^{4}$

This article examines the effect of learning one's HIV status on contraceptive practices in the context of a Phase III multisite randomised controlled trial of the diaphragm for HIV prevention. ${ }^{5}$ 


\section{Methods}

We analysed data from all eligible women who participated in the MIRA trial (trial methods described in detail elsewhere). ${ }^{5}$ Briefly, HIV-negative, non-pregnant, sexually active women who were willing to be randomised to use a diaphragm with lubricant gel, in addition to receiving condoms, safer sex counselling, and curable sexually transmitted infection (STI) testing and treatment and who were willing to return for quarterly visits were enrolled in the study. Women were recruited from family planning, well baby and general health clinics, and through community outreach, in Harare, Zimbabwe and Johannesburg and Durban, South Africa. At baseline (the woman's enrolment visit), women provided detailed demographic data and self-reported contraceptive use during a face-to-face interview. At each quarterly follow-up visit they completed an audio computer-assisted self-interview, participated in a face-to-face interview, and were tested for pregnancy, STIs, and HIV. The open-ended question on contraceptive use was the same at baseline and follow-up namely: "What are you currently doing to prevent pregnancy?". We compared the change in individual responses to this question from baseline to last study visit. Hormonal contraceptive methods and condoms were available free of charge to women participating in the study.

We calculated medians and interquartile ranges (IQRs) for continuous demographic measures and percentages for ordinal measures among women who did and did not become HIV-positive during the MIRA trial (testing algorithm described elsewhere). ${ }^{5}$ In addition, we calculated the frequency of contraceptive use at baseline and at each woman's final visit. Selfreported contraceptive use was grouped into seven categories based on the most effective method reported (in increasing order of effectiveness): no method, 'other' method (including less effective methods like withdrawal, traditional methods, and the diaphragm, which was not promoted in the MIRA trial for contraception though some women reported its use), male or female condoms, progestogen-only oral contraceptives (POP), combined estrogen and progestogen oral contraceptives (COC), injectables, and long-acting methods (including intrauterine devices, implants, and male and female sterilisation). For women who reported using more than one method, we classified their use according to the most effective method (e.g. a woman using COC and condoms for contraception was considered a COC user). We calculated the percentage of women in each group that moved to a more effective method category, used a method in the same category, or moved to a less effective method category from baseline to study exit.

We looked more closely at the group of women who became HIV-positive to see if there were immediate changes in contraceptive use category after learning one's HIV-positive status. We considered using contraception data from the seroconversion visit as our pre-seroconversion data since that information should have been collected prior to rapid HIV testing during each visit, but chose to use the visit prior to exclude the possibility that this might not have occurred in some cases. Screening data (between 2 weeks and 30 days prior to enrolment) were used as the pre-seroconversion data for women who learned they were HIV-positive at their first follow-up visit. Differences in demographic characteristics were evaluated using Mann-Whitney $U$ and Fisher's Exact tests; differences in the median proportion using each category of contraception at baseline and last visit were evaluated using the Jonckheere-Terpstra test; and the change in the pattern of method use was compared using the Chi-square $\left(\chi^{2}\right)$ test. Values of $p<0.05$ were considered statistically significant.

\section{Ethical approval}

The study protocol was reviewed and approved by the University of California at San Francisco Institutional Review Board Committee on Human Research, and by the ethics review committees at all local institutions and collaborating organisations. Written informed consent was obtained from all participants. The trial is registered with ClinicalTrials.gov, number NCT00121459.

\section{Results}

This analysis included 4645 women who remained HIV-negative and 309 women who were infected with HIV during the trial. Table 1 shows the demographic characteristics of each group and contraceptive use at baseline and last visit. The median number of quarterly follow-up visits in both groups was nine. Women who became HIV-positive during the trial were younger, had more lifetime sexual partners, were younger at first sexual intercourse, more likely to be unmarried and not living with a partner or husband, and more likely to live in South Africa. Although significant, many of the differences between the groups were small.

We compared the proportion using each category of contraception between the two groups (Table 1). Though patterns of use of specific methods are different in some cases (data not shown), the proportion of women in each contraception category was similar in the two groups at baseline $(p=0.33)$ as well as at last visit $(p=0.50)$. Injectables, COC, and condoms were the methods most commonly reported. Few women reported using long-acting methods and a substantial minority of women reported using no or an 'other' method. There was no difference in the percentage of women who became pregnant during their study participation by HIV status $(p=0.15)$.

Among both HIV-negative and HIV-positive women, slightly more than one-quarter reported use of a method in a more effective contraceptive category, half did not change method categories, and approximately one-fifth 
changed to a less effective method category between baseline and last visit (Figure 1). The pattern of change was similar between the two groups ( $p=0.81$ ).

We examined contraceptive use among women who became HIV-positive during the study, comparing contraceptive use at the visit prior to and after seroconversion to see if there were short-term changes in response to learning one's HIV status. This analysis included 243 women for whom we had pre- and postseroconversion data. Sixty-six women were excluded because they learned they were HIV-positive at their first and only visit $(n=11)$ or their last visit $(n=53)$, or were missing data $(n=2)$. Reported proportion using each category of contraception was similar at the visit prior to and after learning HIV status: no method 14 (5.76\%) before vs 16 (6.58\%) after, 'other' method 0 vs $2(0.82 \%)$, condoms $74(30.45 \%)$ vs 79 (32.51\%), POP $14(5.76 \%)$ vs $8(3.29 \%)$, COC 40 (16.46\%) vs $37(15.23 \%)$, injectables $87(35.80 \%)$ vs $86(35.39 \%)$, and long-term methods $14(5.76 \%)$ vs $15(6.17 \%)$. Between the visit prior to and after learning HIV-positive status, $17.3 \%$ of women moved to a more effective method category, $18.1 \%$ moved to a less effective method category, and $64.6 \%$ reported using a method in the same category. The largest changes were 12 women who switched from condoms to injectables

Table 1 Participant characteristics by HIV status

\begin{tabular}{|c|c|c|c|}
\hline Characteristic & HIV-negative & HIV-positive & $p^{*}$ \\
\hline \multicolumn{4}{|l|}{ Demographic characteristics $^{\dagger}$} \\
\hline Age in years [median (IQR)] & $27(22-34)$ & $24(21-30)$ & $<0.0001^{\ddagger}$ \\
\hline Years of education [median (IQR)] & $11(8-11)$ & $11(9-11)$ & 0.073 \\
\hline Number of male partners [median (IQR)] & $1 \quad(1-3)$ & $2 \quad(1-3)$ & $<0.0001^{\ddagger}$ \\
\hline Age at first sexual encounter in years [median (IQR)] & $18(16-19)$ & $17(16-19)$ & $0.0012^{\ddagger}$ \\
\hline Marital status $[n(\%)]$ & & & $<0.0001^{\ddagger}$ \\
\hline Married & $2819 \quad(60.7)$ & $122 \quad(39.5)$ & \\
\hline Unmarried & $1824 \quad(39.3)$ & $187 \quad(60.5)$ & \\
\hline Currently living with partner/husband [ $n(\%)]$ & & & $<0.0001^{\ddagger}$ \\
\hline Yes & $3260 \quad(70.2)$ & $145 \quad(46.9)$ & \\
\hline No & $1383 \quad(29.8)$ & $164 \quad(53.1)$ & \\
\hline Country $[n(\%)]$ & & & $<0.0001^{\ddagger}$ \\
\hline Zimbabwe & $2342 \quad(50.4)$ & $114 \quad(36.9)$ & \\
\hline South Africa & $2303 \quad(49.6)$ & $195 \quad(63.1)$ & \\
\hline Contraceptive ${ }^{\S}$ method at baseline $[n(\%)]$ & & & 0.33 \\
\hline None & $(5.4)$ & $16 \quad(5.2)$ & \\
\hline Other & (2.3) & (1.3) & \\
\hline Condoms & $1190 \quad$ (25.6) & $96 \quad$ (31.1) & \\
\hline Progestogen-only pills (POP) & $679 \quad(14.6)$ & $30 \quad(9.7)$ & \\
\hline Combined oral contraceptive pills (COC) & $1022 \quad(22.0)$ & $40 \quad(12.9)$ & \\
\hline Injectables & 1149 (24.7) & $109 \quad(35.3)$ & \\
\hline Long-term methods & $247 \quad(5.3)$ & $14 \quad(4.5)$ & \\
\hline Total & $4645(100.0)$ & $309(100.0)$ & \\
\hline Contraceptive ${ }^{\S}$ method at last visit [ $\left.n(\%)\right]$ & & & 0.50 \\
\hline None & (8.9) & (8.7) & \\
\hline Other & $(0.6)$ & $(1.3)$ & \\
\hline Condoms & $1147 \quad(24.8)$ & $88 \quad(28.5)$ & \\
\hline Progestogen-only pills (POP) & $304 \quad(6.6)$ & $13 \quad(4.2)$ & \\
\hline Combined oral contraceptive pills (COC) & $1236 \quad(26.7)$ & $51 \quad(16.5)$ & \\
\hline Injectables & $1220 \quad(26.4)$ & $108 \quad(35.0)$ & \\
\hline Long-term methods & $283 \quad(6.1)$ & $18 \quad(5.8)$ & \\
\hline Total & $4629(100.0)$ & $309(100.0)$ & \\
\hline Became pregnant during study" $[n(\%)]$ & & & 0.15 \\
\hline Yes & $979 \quad(21.1)$ & $76 \quad$ (24.6) & \\
\hline No & $3666 \quad(78.9)$ & $233 \quad(75.4)$ & \\
\hline
\end{tabular}


and 19 women who switched from injectables to condoms. The overall change in proportion using each category of contraception in the short-term was similar to the results of the analysis of long-term changes, though somewhat more HIV-positive women reported using a method in the same category (64.6\% vs $52.4 \%)$ after seroconversion.

\section{Discussion}

Among women participating in an HIV prevention trial, change in contraceptive method use over the period of study participation was similar among women who remained HIV-negative and women who learned that they were HIV-positive. Although there were some significant demographic differences between the two groups of women, patterns of contraceptive use were similar at study baseline and study end. Learning about HIV-positive status did not appear to significantly change the pattern of contraceptive method use in the short- or long-term, or the probability of switching to a more or less effective method of contraception in this population.

Our results are somewhat different from data in a cohort of HIV-positive women in Malawi where the researchers found that contraceptive use increased 1 week after HIV-positive diagnosis. ${ }^{3}$ We were unable to look at such short-term effects of seroconversion, and contraceptive use returned to rates only slightly higher than baseline at the end of that study. Our results are also somewhat different from those of the study in Kenya and Zambia, which found that HIV-positive women were significantly more likely to use condoms for family planning than HIV-negative women in two of the three sites, though use of other methods was similar

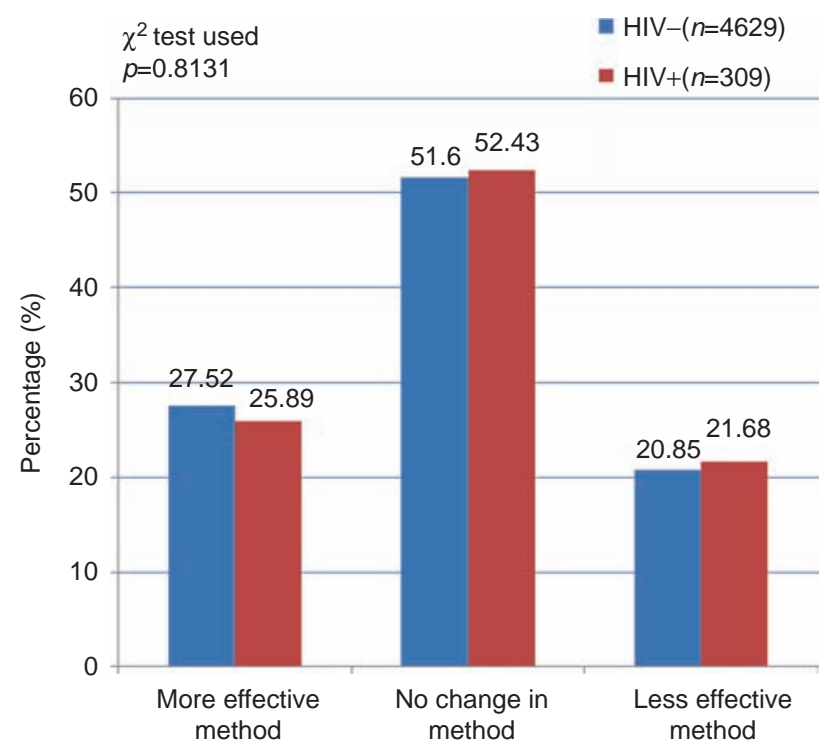

Figure 1 Percentages of women who used methods in more, the same, or less effective contraceptive method categories at last visit compared to baseline by HIV status. between HIV-positive and HIV-negative women in that study. ${ }^{4}$ Our data combined with the other limited evidence available suggest that learning one's HIV-positive status does not lead to dramatic changes in effective contraceptive use although more data are needed on differences in preferred practices, methods considered appropriate and recommended by providers, and whether HIV status affects quality of services and counselling. Qualitative data are needed to provide in-depth information regarding these questions. It is also not clear whether for both HIV-positive and HIV-negative women these and other results reflect limited method choices, and whether the results would be different if a wider range of options was available.

MIRA trial participants were recruited from clinics and offered free hormonal contraception; they started the study with high levels of effective method use and access to these methods was likely to have been easier for women in the general population or in more rural areas. Over the course of our study, approximately onefifth of women, irrespective of serostatus, moved to less effective methods of contraception. At both time periods, use of long-acting contraceptive methods was low. There is a significant need for increased information and access to long-acting contraceptive methods.

This analysis has several limitations. We only collected data on contraceptive use quarterly and therefore are unable to detect more immediate changes post-seroconversion. In addition, we did not collect data on pregnancy intention and therefore are unable to link changes in contraceptive use to potential changes in pregnancy plans, or differences in pregnancy intention between HIV-positive and HIV-negative women. Further, our study products (diaphragm and condoms) are contraceptives (though women in the trial were informed of the unknown contraceptive effectiveness of the diaphragm without spermicide), and the fact that women were asked to use condoms and diaphragms for HIV prevention may have altered some women's contraceptive behaviour, irrespective of their HIV status as suggested by qualitative data collected after the trial (Kacanek D, Dennis A, Sahin-Hodoglugil NN, et al.; unpublished data).

This analysis highlights the need for innovative strategies to increase access and uptake of effective contraceptive methods for women irrespective of HIV status. Contraception, including long-acting methods, should be promoted within international family planning programmes and offered as part of routine care for all women in HIV prevention trials.

Acknowledgements The authors would like to thank the women who participated in this study as well as the larger MIRA staff involved in study implementation and data collection and our Community Advisory Boards. The MIRA trial was funded through a grant from the Bill and Melinda Gates Foundation (\#21082). The data in this article 
were originally presented at Microbicides 2010 Conference in Pittsburg, PA, USA in May 2010.

Funding This work was funded by a grant (\#21082) from the Bill and Melinda Gates Foundation to the University of California, San Francisco, CA, USA.

Competing interests None.

Provenance and peer review Not

commissioned; externally peer reviewed.

\section{References}

1 UNAIDS. Report on the Global AIDS Epidemic. Geneva, Switzerland: UNAIDS, 2010.
2 Population Reference Bureau. Family Planning Worldwide 2008 Data Sheet. 2008. www.prb.org/pdf08/fpds08.pdf [accessed 19 November 2010].

3 Hoffman IF, Martinson FE, Powers KA, et al. The year-long effect of HIV-positive test results on pregnancy intentions, contraceptive use, and pregnancy incidence among Malawian women. J Acquir Immune Defic Syndr 2008;47:477-483.

4 Rutenberg N, Baek C. Review of Field Experiences: Integration of Family Planning and PMTCT Services. New York, NY: Population Council, 2004.

5 Padian NS, van der Straten A, Ramjee G, et al.; MIRA Team. Diaphragm and lubricant gel for prevention of HIV acquisition in southern African women: a randomised controlled trial. Lancet 2007;370:251-261. 